\title{
Thriving in a changing world - opportunities and challenges for private health insurance companies
}

The health insurance market in Poland reflects global trends - such as the rising awareness of personal health impact on quality of life. As a consequence, the health insurance market has seen substantial growth during the last years, which is forecasted to continue at over 20 percent more than life or P\&C insurance globally. However, private health insurance has not yet unlocked its full potential. During the last few years, the growth of the private healthcare insurance segment has been outgrowing the public segment (see exhibit 1). This is due to three main drivers, which reveal excellent market opportunities for $\mathrm{PHI}$ in Poland:

- Speed of service. At the moment service is very slow, presenting a considerable opportunity as this is the most important factor for customer satisfaction

- Treatment quality. There are less doctors per capita in Poland than other European countries, which could be the main reason that treatment quality is perceived as poor

- Inpatient services. Many companies are focused on outpatient coverage, which results in a lack of inpatient coverage and unmet customer needs: covering more inpatient services than public providers could be an attractive option

Exhibit 1: Globally, health insurance outperforms life and P\&C. In Poland, PHI is outgrowing public payors

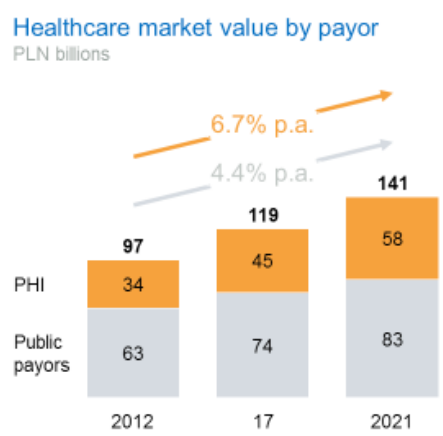

Still the majority of private health expenditure is out-of-pocket. 


\section{Addressing customer satisfaction is an excellent market opportunity}

The rise in private healthcare insurance is likely due to patients' dissatisfaction with the Polish public healthcare system. Almost two-thirds of the population describe the healthcare system as being "bad," whereas the EU average is below one-third. Over 70 percent of Polish patients even believe that the hospital could harm them. These perceptions arise especially when considering that Polish healthcare expenditure per capita is comparatively low to other European countries (see exhibit 2).

Exhibit 2: Polish healthcare is currently experiencing challenges, which drives the rise of $\mathrm{PHI}$
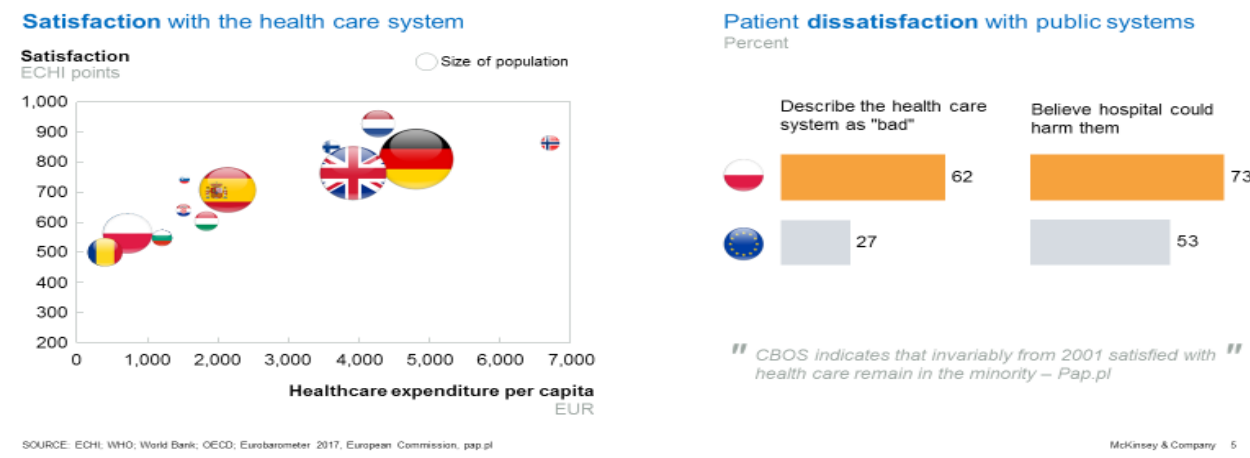

Long waiting times are the main contributor to this low level of satisfaction with the public healthcare system. The Organization for Economic Co-operation and Development has shown that the average waiting times from specialist assessment to treatment can be more than 300 percent longer in Poland than in the EU on average.

Responding to the unmet patient needs identified above, and especially the long waiting times, can translate into quick wins for PHI. By offering quicker service, better quality, and comprehensive choice, companies can maximize the value for the customer - which will pay off.

\section{Increasing business risks require new business models}

Healthcare companies should make sure not to forget about other opportunities while they focus on customer satisfaction. An increasingly complex world environment and continuous demographic change necessitate flexibility in the development of new business models.

As the population ages and medicine improves, there is a shift towards more predictable risks. WHO forecasts that communicable diseases will have decreased by approximately 30 percent in 2030 as compared to 2005 levels. However, the number one cause of non-natural deaths, chronic diseases (including cancer), will become more prevalent. This shift has an immediate impact on:

- Capability requirements

Various players in the healthcare industry are already working towards an efficient health system that uses digital models. We anticipate an increasing use of advanced analytics and big 
data driving predictive analyses and helping address unmet patient needs. This will generate new capability requirements for healthcare insurers

- Product range

While most health insurers still offer a relatively basic product portfolio, more advanced products are on their way. A lot of medtech start-ups and companies are already focusing on the customer journey and aim to offer a patient health journey that is fully integrated

- Risk management

Payors are spreading their rising medical risk by offering incentives to both the provider and the consumer (see exhibit 3). While they are increasing the percentage of outcome-based payment, they are also sharing the risk (and the profit) with healthcare providers. Simultaneously, they reward healthy customers by offering them premiums and decreasing the risk of illness.

Exhibit 3: Risk sharing along the value chain is important in order to cope with increasing risk, ensure system efficiency, and improve patient outcomes

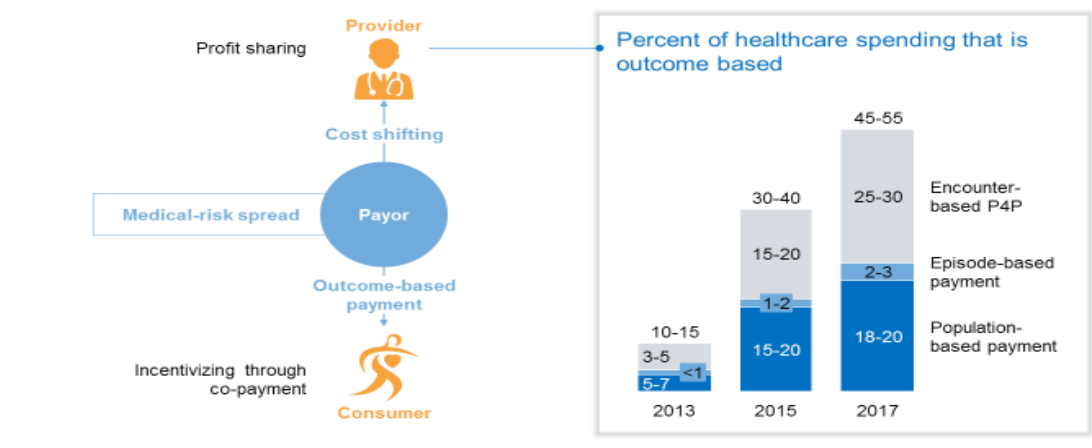

\section{Technology and digital reshaping unlocks opportunities}

The future is never free from surprises, and classical business models are evolving along with the rapidly changing environment. We see three key areas to excel in for success.

\section{- Area 1: Strengthening the relationship with consumers by offering better products}

In a complex and volatile world, customers are seeking high-quality, convenient, and transparent solutions. There are opportunities to win these customers by offering integrated services throughout the customer journey.

Distinct value propositions are blurring into a complete selection of targeted/individualized services of providers, payors, pharma and medical devices. The first step in integrating services is for payors to position themselves as "partners" for customers, who then feel understood and sense the desire to have their experience improved.

While companies start to broaden their offerings, and enter new value pools, like wellness, we see that customer experience and satisfaction is mostly linked to digitalization of a segment. 
This assumption is reinforced by the fact that healthcare consumers are quickly adopting digital solutions and crave for more digital offerings. According to McKinsey Consumer Health Insights survey:

- $63 \%$ believe scheduling a medical appointment online is more effective than by phone

- $\quad>70 \%$ of consumers are now engaging in digital channels for medical education

- $63 \%$ of consumers would prefer a single app that could hold all of their data (including medical records]

However, the overall healthcare industry still lags behind other industries (e.g., overall customer satisfaction 2017 in Health $76 \%$ compared to $82 \%$ of leading E-commerce segment).

Thus, digital solutions offer quick wins for payors and emerging companies as they provide a large platform for customer interaction. There is already a variety of start-ups closing gaps of traditional provider-payor offerings. But there is a high potential to grasp for innovative solutions and the development of integrated ecosystems of all kind of health-related services. Smart data can help to better understand customer desires and respond in a more personalized way.

\section{- Area 2: Generating value for the entire system and improving outcomes}

In a world of uncertainty, risk management is key to success. For PHI, there are opportunities to leverage risk prediction and reallocate risk along the value chain to generate value and improve outcomes for patients. However, increased efficiency of this field is required to excel. The time of full indemnity plans are over. Payors should not take full risk covering all the costs of health provision. They must evaluate their business models upon risk sharing among them, providers and patients. Aligning incentives while maintaining best patient outcomes can be made possible with e.g., high-deductible health plans, where members have higher co-payments, when activating the plan. Moreover, specific partnerships (e.g., US partnerships) of providers and payors can create additional value for the health system through efficiency gain and better risk management as well.

Risk management is closely linked to smart processing of data and excelling on digitalization level is equally crucial to win in this field. Consequently, the private healthcare industry (both private providers and payors] is increasingly using advanced analytics to predict and manage cost and risk as well as to shine in claims handling and fraud identification.

\section{- Area 3: Tapping into new value pools by reinventing business models}

A rapidly changing world demands non-traditional and agile business models. There are opportunities to win in this environment through smart partnering inside but also outside the healthcare space and broadening of value propositions.

As described above, payors need new business models in order to respond to customer needs and secure themselves against risks. The market gets more competitive and as new players are entering with disrupting business models, traditional big players realize capability gaps. Players along the value chain must head for new shores and embrace partnering to integrate and implement new business models. The integrated partnership follows a carefully balanced approach of creating new offerings while maintaining own brand recognition. This might require 
a re-specialization for companies and entering niches to increase competitiveness by differentiation (e.g., move into healthcare data analytics). However, partnership now seems to be more important than ever before to stay relevant in the market by keeping new entrants (e.g., tech companies] at distance.

There are three possible kinds of partnership for payors

- Horizontal

Horizonal integration derive from the inside and include payor-to-payor or provider-toprovider partnerships. Players should use this strategy to tuck-in breadth or depth to their geography, services, or customer segments.

- Vertical

Vertical integration includes payor-to-provider mergers. This inside-out partnership can serve for strategic effects to extract efficiencies along the supply chain, allowing for better outcomes and lower prices for consumers.

\section{- External (Innovation)}

External integration includes partnerships of companies from outside the healthcare system. The outside-in relationship gives the ability to joint forces and strategically drive down healthcare costs for employees and employers.

However, there is another point for attention. In order to equally partner with a provider, its trends have to be understood completely. Our research has shown that there are 5 distinct characteristics which determine the new archetype of a successful future provider.

\section{- Increase value-based care}

Higher quality is achieved by supporting patients - at the required place and time. Care management includes a decent number of HCPs, who respond to individual patient needs. Investing into smart data and understanding customers is advantageous for providers. They will likely link in a vertical partnership with the payor to seize this opportunity and achieve the necessary scale for good service through horizontal partnerships

\section{- Become a high-efficiency operating company}

Scale also reduces cost - which is essential for an efficiently operating company. Thus, providers will consider not only horizontal mergers but also national shared service offerings (e.g., purchasing, revenue cycle, hospital operations] to expand acute care regionally or nationally.

- Expand regionally dense and geographically isolated acute care Providers will leverage their traditional market position to gain strength against payors in a FFS environment. For instance, they can build or acquire in-market acute care.

\section{- Acquire across continuum of care}

The horizontal partnership trend will drive providers to acquire across continuum of care to take control of entire patient lifecycle. They will mostly focus on building or acquiring non-acute providers (e.g., physician groups, urgent care, post-acute). 


\section{- Differentiated services and branding}

The trend of differentiation also takes place in provider universe, which payors can profit from. Providers can leverage assets that cannot be easily replicated, e.g., premium brand, unique services (NICU1, trauma). By partnering with these specific providers, payors can offer unique services to their customers.

Understanding the key three areas of success along with the simultaneous evolvement of other system components (like partners) is crucial for payors. However, it is equally important to individually prioritize areas of action and focusing on establishing a brand.

$\mathrm{PHI}$ can fully capture rising opportunities in Poland by continuing to develop health insurance products that satisfy the unmet needs of customers to the maximum extent possible. Only higher service quality, more convenience, and better medical outcomes will drive customers to the PHI segment and ensure that it reaches its full potential.

DR. TOBIAS SCHNEIDER is an Associate Partner in McKinsey's Cologne office. He is a member of the German Healthcare Practice. He possesses extensive expertise in international health insurance and private health insurance markets and has worked on topics such as strategy, improvement of operations, cost containment, organization, and data and analytics. Tobias is a licensed physician. He studied medicine at the Technical University of Munich, Harvard Medical School, Boston, and Johns-Hopkins Medical School, Baltimore. Prior to joining McKinsey, Tobias conducted medical research focusing on mass spectrometric and chromatographic protein analysis, embryology, epigenetics, and pluripotency as well as stem cell research. Tobias has also worked as a physician in Switzerland. 